Pacific Journal of Mathematics

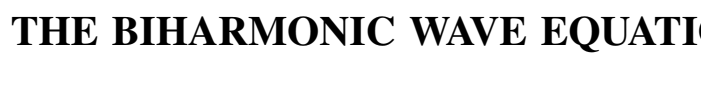




\section{ON THE BIHARMONIC WAVE EQUATION}

\section{ERNEST L. ROETMAN}

Under appropriate restrictions of material and motion the equation of motion for a vibrating elastic bar is $\left(\partial_{x}^{4}+\partial_{t}^{2}\right) u=0$. Because of its mechanical importance, there is a large literature devoted to the eigenvalue problem for this equation but solutions of boundary value problems for the equation itself seem to have been ignored. It appears that Pini was the first to seek a solution in terms of integrals analogous to thermal potentials. Like Pini, we use a fundamental solution very similar to that of the heat kernel to obtain potential terms which lead to a system of integral equations. While Pini uses Laplace transforms to obtain solutions to the integral equations, we observe that the problem may be reduced to one integral equation of a complex valued function, $f=a+\lambda k * \bar{f}$, effecting a significant simplification.

Along the way, we obtain, by reduction to Abel integral equations, a general method of solving semi-infinite problems which can solve boundary value problems not available to Fourier transforms, the technique presently used.

The first appendix is a justification of the change of order of integration for a key iterated integral; the computation of some important integrals is given in the second appendix.

2. The fundamental solutions. By standard Fourier transform techniques, one finds that a fundamental solution for the equation

$$
\left(\partial_{x}^{4}+\partial_{t}^{2}\right) u=0
$$

is

$$
K(x, t)=-\pi^{-1 / 2} t^{1-1 / 2} \exp \left(\frac{i x^{2}}{4 t}+i \frac{\pi}{4}\right)
$$

We also define

$$
C(x, t)=\operatorname{Re} K(x, t), \quad S(x, t)=\operatorname{Im} K(x, t) .
$$

We obtain by straightforward computation:

$$
\begin{gathered}
\partial_{x} K=\frac{i x}{2 t} K, \\
\left(\partial_{x}^{2}+i \partial_{t}\right) K=0, \\
\left(\partial_{x}^{2}-i \partial_{t}\right) \bar{K}=0,
\end{gathered}
$$


and hence

$$
\left(\partial_{x}^{4}+\partial_{t}^{2}\right)\left\{\begin{array}{l}
C \\
S
\end{array}\right\}=0
$$

It is convenient to set

$$
K_{1}(x, t)=\int_{0}^{t} K(x, s) d s
$$

thus

$$
K_{1}(x, t)=\frac{a x}{2} \int_{h}^{\infty} s^{-3 / 2} e^{i s} d s
$$

where

$$
a=-\pi^{-1 / 2} e^{i \pi / 4} \quad \text { and } \quad h=\frac{x^{2}}{4 t} .
$$

Then

$$
\begin{gathered}
\partial_{x} K_{1}=\frac{1}{x} K_{1}-\frac{2 t}{x} K, \\
\partial_{x}^{2} K_{1}=-i K \\
\left(\partial_{x}^{2}+i \partial_{t}\right) K_{1}=0 .
\end{gathered}
$$

3. Semi-infinite bar. We consider now the problem of the semiinfinite bar; that is, we seek a function $u(x, t)$ on $D=\{0<x\} \times(0, T)$ such that

$$
\left(\partial_{x}^{4}+\partial_{t}^{2}\right) u=0,
$$

and in the limit

$$
\begin{gathered}
u(x, 0)=\partial_{t} u(x, 0)=0, \\
u(0+, t)=a(t) \\
\partial_{x} u(0+, t)=b(t)
\end{gathered}
$$

where the conditions on the functions $a$ and $b$ will be determined presently.

We try a solution in the form

$$
u(x, t)=\int_{0}^{t}[C(x, t-s) \varphi(s)+S(x, t-s) \psi(s)] d s .
$$

To relate $\varphi$ and $\psi$ to $a$ and $b$, we consider

$$
U(x, t ; \varphi)=\int_{0}^{t} K(x, t-s) \varphi(s) d s .
$$


The next two theorems are essentially contained in [3] but for completeness we include them here. (We use BV and CBV to mean respectively the classes of functions of bounded variation and continuous and of bounded variation.)

Theorem 1. If $\varphi$ is $\mathrm{BV}$ on $[0, T]$, then

$$
\left(\partial_{x}^{2}+i \partial_{t}\right) U=0
$$

on $D$.

Proof. By integration by parts

$$
U=\varphi(0) K_{1}+\int_{0}^{t} K_{1}(x, t-s) d \varphi(s)
$$

so that by (11)

$$
\partial_{x}^{2} U=-i \varphi(0) K(x, t)+\int_{0}^{t} K(x, t-s) d \varphi(s) .
$$

Differentiation of (18) with respect to $t$ and comparison with (19) completes the proof. Defining

$$
\begin{aligned}
& u_{c}(x, t ; \varphi)=\operatorname{Re} U(x, t ; \varphi) \\
& u_{s}(x, t ; \varphi)=\operatorname{Im} U,
\end{aligned}
$$

we have

Corollary. If $\varphi \in C^{1}$ and $\varphi^{\prime} \in \mathrm{BV}$ on $[0, T]$, then $u_{c}$ and $u_{s}$ satisfy (13).

Proof. Since (19) can be written as

$$
\partial_{x}^{2} U=-i \varphi(0) K(x, t)-i U\left(x, t ; \varphi^{\prime}\right),
$$

we can apply Theorem 1 again.

Theorem 2. If $\varphi \in \operatorname{CBV}([0, T])$, then

$$
\begin{gathered}
\lim _{\substack{x \rightarrow 0 \pm \\
t \rightarrow t_{0}}} U(x, t ; \varphi)=-\pi^{-1 / 2} e^{i \pi / 4} \int_{0}^{t_{0}}\left(t_{0}-s\right)^{-1 / 2} \varphi(s) d s, \\
\lim _{\substack{x \rightarrow 0 \pm \\
x \rightarrow t_{0}}} \partial_{x} U(x, t ; \varphi)= \pm \varphi\left(t_{0}\right)
\end{gathered}
$$

on $(0, T)$, and

$$
\lim _{\substack{t \rightarrow 0+\\ x \rightarrow x_{0}}} U=\lim _{\substack{t \rightarrow 0+\\ x \rightarrow x_{0} \neq 0}} \partial_{x} U=0 .
$$


Proof. Equation (21) and the first equality in (23) follow easily by standard arguments. That

$$
\int_{0}^{t} \partial_{x} K(x, t-s) \varphi(s) d s
$$

exists for all $x>0$ follows immediately from

$$
\left|\int_{t_{1}}^{t_{2}} \partial_{x} K \varphi d s\right| \leqq\left(\left|\varphi\left(t_{1}\right)\right|+V\left(\varphi ; t_{1}, t_{2}\right)\right) M\left(t_{1}, t_{2}\right),
$$

where

$$
M\left(t_{1}, t_{2}\right)=\sup \left|\int_{w}^{z} \partial_{x} K d s\right|, \quad t_{1} \leqq w<z \leqq t_{2}
$$

(see [2, p. 623]) and, since the integral exists, goes to zero as $t_{1}, t_{2} \rightarrow t$. (The second part of (23) obtains from $M(0, t) \rightarrow 0$ as $t \rightarrow 0$.) On the other hand, by integration by parts,

$$
\begin{aligned}
\left|\int_{w}^{z} \partial_{x} K d s\right|< & -\frac{1}{2 \sqrt{\pi}} \frac{1}{|x|}\left\{2\left[z^{1 / 2}-w^{1 / 2}\right]\right. \\
& \left.+\left|\int_{w}^{z} \exp \left(\frac{i x^{2}}{4 s}\right) s^{-1 / 2} d s\right|\right\} \leqq \frac{1}{\sqrt{\pi}} \frac{1}{|x|}\left[z^{1 / 2}-w^{1 / 2}\right]
\end{aligned}
$$

so that the convergence is uniform with respect to $x$ for $|x| \geqq \delta>0$. Therefore

$$
U_{x}(x, t ; \varphi)=\int_{0}^{t} K_{x}(x, t-s) \varphi(s) d s
$$

For $\varphi=1$,

$$
U_{x}(x, t ; 1)=\int_{0}^{t} K_{x}(x, s) d s=-\frac{e^{i \pi / 4}}{2 \sqrt{\pi}} i x \int_{0}^{t} s^{-3 / 2} \exp \left(\frac{i x^{2}}{4 s}\right) d s
$$

which through a change of variable becomes

$$
U_{x}(x, t ; 1)=-\frac{i e^{i \pi / 4}}{\sqrt{\pi}} \operatorname{sgn}(x) \int_{h}^{\infty} m^{-1 / 2} e^{\imath m} d m
$$

where $h=x^{2} / 4 t$. Since

$$
\begin{gathered}
\lim _{\substack{x \rightarrow 0 \pm \\
t \rightarrow t_{0}}} h=0, \\
\lim _{\substack{x \rightarrow 0 \pm \\
t \rightarrow t_{0}}} U_{x}(x, t ; 1)= \pm 1 .
\end{gathered}
$$

Now, for $x>0$ 


$$
\begin{aligned}
& \int_{11}^{t} \partial_{x} K(x, t-s)\left[\varphi(s)-\varphi\left(t_{0}\right)\right] d s \\
& =\left(\int_{0}^{t-\delta}+\int_{t-\delta}^{t}\right) \partial_{x} K\left[\varphi(s)-\varphi\left(t_{0}\right)\right] d s \\
& \quad=I_{1}+I_{2},
\end{aligned}
$$

and

$$
\left|I_{1}\right| \leqq \frac{|x|}{\sqrt{\pi}} \sup \left|\varphi(s)-\varphi\left(t_{0}\right)\right|\left(\delta^{-1 / 2}-t^{-1 / 2}\right)
$$

which goes to zero as $t \rightarrow t_{0}$ and $x \rightarrow 0+$. Also,

$$
\left|I_{2}\right| \leqq\left\{\sup _{t-\delta \leqq s \leqq t}\left|\mathcal{P}(s)-\phi\left(t_{0}\right)\right|+V\left(\varphi(s)-\varphi\left(t_{0}\right) ; t-\delta, t\right)\right\} M(x)
$$

where

$$
M(x)=\sup \left|\int_{t_{1}}^{t_{2}} \partial_{x} K(x, t-s) d s\right|,
$$

$t-\delta \leqq t_{1}<t_{2} \leqq t . \quad$ But

$$
\left|\int_{t_{2}}^{t_{2}} \partial_{x} K d s\right|=\frac{1}{\pi}\left|\int_{h\left(t_{1}\right)}^{h\left(t_{2}\right)} m^{-1 / 2} e^{i m} d m\right|
$$

where $h(s)=x^{2} / 4(t-s)$, and since the limits of integration are always positive and the integral on $(0, \infty)$ exists, the last integral is uniformly bounded in $x$ and $t$, i.e. $M(x) \leqq M_{1}$. Therefore,

$$
\left|I_{2}\right| \leqq M_{1}\left\{\sup \left|\varphi(s)-\varphi\left(t_{0}\right)\right|+V\left(\varphi(s)-\varphi\left(t_{0}\right) ;(t-\delta, t)\right)\right\} .
$$

Thus, for $t$ sufficiently close to $t_{0}$ and $\delta$ so small that $t-\delta$ is also close to $t_{0}$, the continuity of $\varphi$ and of its variation implies that $\left|I_{2}\right|$ is small which completes the proof.

We shall later find it necessary to extend these theorems for an important special case which is not contained in the above hypotheses. We can show that our theorems do not hold without the BV requirement, but as we shall see, BV is not necessary; thus, the present conditions are not the most natural for the kernel in question.

From the preceding we see that the conditions (14) are satisfied and that the boundary conditions (15) must be related to the density functions $\varphi$ and $\psi$ by

$$
\begin{aligned}
-\frac{1}{\sqrt{2 \pi}} \int_{0}^{t}(t-s)^{-1 / 2} \varphi(s) d s & -\frac{1}{\sqrt{2 \pi}} \int_{0}^{t}(t-s)^{-1 / 2} \psi(s) d s=a(t) \\
\varphi(t) & =b(t) .
\end{aligned}
$$

We then have 


$$
\begin{gathered}
\varphi(t)=b(t) \\
\psi(t)=-\sqrt{2} \frac{d}{d t} \int_{0}^{t}(t-s)^{-1 / 2} a(s) d s-b(t) .
\end{gathered}
$$

That is, we have proved that:

THEOREM 3. If $b(t)$ and $d / d t \int_{0}^{t}(t-s)^{-1 / 2} a(s) d s$ have first derivatives which are CBV[0,T], then (16) satisfies (13) with conditions (14) and (15) where $\varphi$ and $\psi$ are given by (27).

4. The finite bar problem. We consider next the problem of finding a function $u(x, t)$ on the domain

$$
D=\{0<x<2\} \times(0, T)
$$

which satisfies

$$
\left(\partial_{x}^{4}+\partial_{t}^{2}\right) u=0 \text { in } D
$$

with

$$
u(x, 0+)=\partial_{t} u(x, 0+)=0
$$

and

$$
\begin{array}{cl}
u(0+, t)=a_{1}(t), & u(2-, t)=a_{2}(t) \\
\partial_{x} u(0+, t)=b_{1}(t), & \partial_{x} u(2-, t)=b_{2}(t)
\end{array}
$$

for $t>0$.

We seek a solution in the form

$$
\begin{aligned}
u(x, t)= & u_{c}\left(x, t ; \varphi_{1}\right)+u_{s}\left(x, t ; \psi_{1}\right) \\
& +u_{c}\left(2-x, t ; \varphi_{2}\right)+u_{2}\left(2-x, t ; \psi_{2}\right) .
\end{aligned}
$$

We observe that, by the corollary to Theorem 1 , if $\varphi_{i}$ and $\psi_{i}(1=1,2)$ have CBV derivatives, then (30) satisfies (28) and, by Theorem 2, that (30) satisfies the initial conditions and finally that there hold the relations

$$
2^{-1 / 2} I^{1 / 2} \varphi_{1}+2^{-1 / 2} I^{1 / 2} \psi_{1}+\left\{t^{-1 / 2} \cos \left(\frac{1}{t}\right)+\frac{\pi}{4}\right\} * \varphi_{2}
$$

$$
+\left\{t^{-1 / 2} \sin \left(\frac{1}{t}+\frac{\pi}{4}\right)\right\} * \psi_{2}=-a_{1}
$$

$$
\varphi_{1}+2\left\{t^{-3 / 2} \sin \left(\frac{1}{t}+\frac{\pi}{4}\right)\right\} * \varphi_{2}-2\left\{t^{-3 / 2} \cos \left(\frac{1}{t}+\frac{\pi}{4}\right)\right\} * \varphi_{2}=b_{1}
$$




$$
\begin{gathered}
\left\{t^{-1 / 2} \cos \left(\frac{1}{t}+\frac{\pi}{4}\right)\right\} * \varphi_{1}+\left\{t^{-1 / 2} \sin \left(\frac{1}{t}+\frac{\pi}{4}\right) * \psi_{1}\right. \\
+2^{-1 / 2} I^{1 / 2} \varphi_{2}+2^{-1 / 2} I^{1 / 2} \psi_{2}=-a_{2} \\
\text { (31 }) \quad 2\left\{t^{-3 / 2} \sin \left(\frac{1}{t}+\frac{\pi}{4}\right)\right\} * \varphi_{1}-2\left\{t^{-3 / 2} \cos \left(\frac{1}{t}+\frac{\pi}{4}\right)\right\} * \psi_{1}+\varphi_{2}=-b_{2},
\end{gathered}
$$
where

$$
f * g=\int_{0}^{t} f(t-s) g(s) d s
$$

and

$$
I^{\alpha} \varphi(t)=(\Gamma(\alpha))^{-1} \int_{0}^{t}(t-s)^{\alpha-1} \varphi(s) d s .
$$

This system is equivalent to that found by Pini [3, p. 101].

For convenience we define operators

$$
\begin{aligned}
& E^{\alpha} \varphi=\frac{e^{i \pi / 4}}{\Gamma(\alpha)}\left\{t^{\alpha-1} \exp \left(i t^{-1}\right)\right\} * \varphi \\
& \widetilde{E}^{\alpha} \varphi=\frac{1}{\Gamma(\alpha)}\left\{t^{\alpha-1} \exp \left(i t^{-1}\right)\right\} * \varphi
\end{aligned}
$$

and

$$
C^{\alpha}+i S^{\alpha}=E^{\alpha}, \quad \widetilde{C}^{\alpha}+i \widetilde{S}^{\alpha}=\widetilde{E}^{\alpha},
$$

so that the system (31) can be written

$$
\begin{aligned}
2^{-1 / 2} I^{1 / 2} \varphi_{1}+2^{-1 / 2} I^{1 / 2} \psi_{1}+C^{1 / 2} \varphi_{2}-S^{1 / 2} \psi_{2} & =-a_{1} \\
I^{0} \varphi_{1}+2 S^{-1 / 2} \varphi_{2}-2 C^{-1 / 2} \psi_{2} & =b_{1} \\
C^{1 / 2} \varphi_{1}+S^{1 / 2} \psi_{1}+2^{-1 / 2} I^{1 / 2} \varphi_{2}+2^{-1 / 2} I^{1 / 2} \psi_{2} & =-a_{2} \\
2 S^{-1 / 2} \varphi_{1}-2 C^{-1 / 2} \psi_{1}+\varphi_{2} & =-b_{2}
\end{aligned}
$$

Adding and subtracting the first and third equations and the second and fourth equations respectively and setting

$$
\begin{aligned}
\varphi_{1}+\varphi_{2} & =f_{1} & \psi_{1}+\psi_{2} & =g_{1} \\
\varphi_{1}-\varphi_{2} & =f_{2} & \psi_{1}-\psi_{2} & =g_{2} \\
a_{1}+a_{2} & =A_{1} & b_{1}-b_{2} & =B_{1} \\
a_{1}-a_{2} & =A_{2} & b_{1}+b_{2} & =B_{2},
\end{aligned}
$$

we obtain two systems of two equations each:

$$
\begin{aligned}
-2^{-1 / 2} I^{1 / 2} f_{1}-2^{-1 / 2} I^{1 / 2} g_{1}-C^{1 / 2} f_{1}-S^{1 / 2} g_{1} & =A_{1} \\
f_{1}+2 S^{-1 / 2} f_{1}-2 C^{-1 / 2} g_{1} & =B_{1}
\end{aligned}
$$


and

$$
\begin{aligned}
-2^{-1 / 2} I^{1 / 2} f_{2}-2^{-1 / 2} I^{1 / 2} g_{2}+C^{1 / 2} f_{2}+S^{1 / 2} g_{2} & =A_{2} \\
f_{2}-2 S^{-1 / 2} f_{2}+2 C^{-1 / 2} g_{2} & =B_{2}
\end{aligned}
$$

Defining operator matrices

$$
M=\left[\begin{array}{cc}
-2^{-1 / 2} I^{1 / 2} & -2^{-1 / 2} I^{1 / 2} \\
I^{0} & 0
\end{array}\right]
$$

and

$$
N_{1}=\left[\begin{array}{cc}
C^{1 / 2} & S^{1 / 2} \\
-2 C^{-1 / 2} & 2 C^{-1 / 2}
\end{array}\right],
$$

we can write (26) and (27) respectively as

$$
M\left[\begin{array}{l}
\varphi \\
\psi
\end{array}\right]=\left[\begin{array}{l}
a \\
b
\end{array}\right]
$$

and

$$
\left[\begin{array}{l}
\varphi \\
\psi
\end{array}\right]=M^{-1}\left[\begin{array}{l}
a \\
b
\end{array}\right]
$$

where

$$
M^{-1}=\left[\begin{array}{cc}
0 & I^{0} \\
-2^{1 / 2} I^{-1 / 2} & I^{0}
\end{array}\right]
$$

Hence, (36) can be written as

$$
\left[\begin{array}{l}
f_{1} \\
g_{1}
\end{array}\right]=\left[\begin{array}{l}
C_{1} \\
D_{1}
\end{array}\right]+M^{-1} N_{1}\left[\begin{array}{l}
f_{1} \\
g_{1}
\end{array}\right]
$$

with

$$
\left[\begin{array}{l}
C_{1} \\
D_{1}
\end{array}\right]=M^{-1}\left[\begin{array}{l}
A_{1} \\
B_{1}
\end{array}\right]
$$

To simplify $M^{-1} N_{1}$, we prove:

Lemma. If $\phi$ is $\mathrm{CBV}[0, T]$, then

$$
I^{-1 / 2} E^{1 / 2} \varphi=-2 \widetilde{E}^{-1 / 2} \varphi .
$$

Proof. Consider

$$
H(t)=\frac{1}{\pi} \int_{0}^{t} \int_{0}^{s} \varphi(r) \exp (i /(s-r))[(t-s)(s-r)]^{-1 / 2} d r d s
$$


which is a absolutely integrable and, hence, can be written as

$$
\begin{aligned}
H(t) & =\frac{1}{\pi} \int_{0}^{t} d r \varphi(r) \int_{r}^{t} \exp (i /(s-r))[(t-s)(s-r)]^{-1 / 2} d s \\
& =\int_{0}^{t} \varphi(r) \operatorname{Erf} c\left(e^{-i \pi / 4}(t-r)^{-1 / 2}\right) d r .
\end{aligned}
$$

Observe that

$$
\frac{d}{d t} \operatorname{Erfc}\left(e^{-i \pi / 4}(t-r)^{-1 / 2}\right)=\frac{1}{2} \sqrt{\pi} \partial_{x} K(2, t-r)
$$

and define

$$
F(t)=\frac{1}{2} \sqrt{\pi} \partial_{x} K(2, t) * \varphi
$$

Then,

$$
\begin{aligned}
t^{-1}[H(t+r)-H(t)]-F(t) \\
=\int_{0}^{t-\delta} \varphi(r)\left\{\tau^{-1}\left[E\left((t+\tau-r)^{-1 / 2}\right)-E\left((t-r)^{-1 / 2}\right)\right]\right. \\
\left.\quad-\frac{1}{2} \pi^{3 / 2} \partial_{x} K(2, t-r)\right\} d r \\
\quad+\tau^{-1} \int_{t-\delta}^{t+\tau} \varphi(r) E\left((t+\tau-r)^{1 / 2}\right) d r \\
\quad-\tau^{-1} \int_{t-\delta}^{t} \varphi(\tau) E\left((t-r)^{-1 / 2}\right) d r \\
=I_{1}+I_{2}-I_{3},
\end{aligned}
$$

where $E(s)=\operatorname{Erfc}\left(e^{-i \pi / 4} s\right)$ and $\delta$ is to be chosen.

If $\tau>0$, we write

$$
\begin{aligned}
I_{2}-I_{3}= & \left.\int_{0}^{\delta} \varphi(t-p) \tau^{-1}\left[E(p+\tau)^{-1 / 2}\right)-E\left(p^{-1 / 2}\right)\right] d p \\
& +\tau^{-1} \int_{0}^{\tau} \varphi(t+\tau-p) E\left(p^{-1 / 2}\right) d p=J_{1}+J_{2} .
\end{aligned}
$$

By the properties of the complimentary error functions,

$$
E\left((p+\tau)^{-1 / 2}\right)-E\left(p^{-1 / 2}\right)=2 e^{i \pi / 4} \int_{p}^{p+\tau} e^{-i / n} n^{-3 / 2} d n,
$$

whence,

$$
\left|J_{1}\right| \leqq 2\{\sup (|\varphi| ;[0, T])+V(\varphi ;[0, T])\} M(\tau, \delta)
$$

where

$$
M(\tau, \delta)=\sup _{0 \leqq w<z \leqq \delta}\left|\int_{w}^{z}\left(\tau^{-1} \int_{p}^{p+\tau} e^{-i / n} n^{-3 / 2} d n\right) d p\right|
$$


Integrating the inside integral by parts and then integrating by parts with respect to $p$, we obtain

$$
\begin{aligned}
\int_{w}^{z}(\cdots) d p= & -i\left[\tau^{-1}\left(e^{-i /(p+\tau)}(p+\tau)^{5 / 2}-e^{-i / p} p^{5 / 2}\right)\right]_{w}^{z} \\
& +\frac{5}{2} i \int_{w}^{z} \tau^{-1}\left(e^{-i /(p+\tau)}(p+\tau)^{3 / 2}-e^{-i / p} p^{3 / 2}\right) d p \\
& +\frac{1}{2} i \int_{w}^{z}\left(\tau^{-1} \int_{p}^{p+\tau} e^{i / n} n^{-1 / 2} d n\right) d p
\end{aligned}
$$

Now then, we are justified in taking the limit as $\tau \rightarrow 0$ to obtain

$$
\begin{aligned}
\varlimsup_{\tau \rightarrow 0} M(\tau, \delta) \leqq & \sup \mid\left[e^{-i / p}\left(i p^{1 / 2}+5 / 2 p^{3 / 2}\right)\right]_{w}^{z} \\
& -5 / 2 \int_{w}^{z} e^{-i p}\left(i p^{-1 / 2}+3 / 2 p^{1 / 2}\right) d p \\
& -1 / 2 \int_{w}^{z} e^{-i / p} p^{-1 / 2} d p \mid \\
\leqq & A \delta^{1 / 2} \quad \text { if } \delta<1 .
\end{aligned}
$$

On the other hand,

$$
\begin{aligned}
\left|J_{2}\right| & \leqq \sup (|\varphi| ;[0, T])\left(\tau^{-1} \int_{0}^{\tau}\left|E\left(p^{-1 / 2}\right)\right| d p\right) \\
& \leqq B \tau^{-1} \int_{0}^{\tau}\left[p^{+1 / 2}+O\left(p^{3 / 2}\right)\right] d p \\
& \leqq B\left\{\tau^{1 / 2}+O\left(\tau^{3 / 2}\right)\right\}
\end{aligned}
$$

which goes to zero with $\tau$. Thus, choosing $\delta$ such that

$$
\varlimsup_{\tau \rightarrow 0}\left|J_{1}\right|<\varepsilon
$$

and holding it fixed, we have

$$
\varlimsup_{\tau \rightarrow 0}\left|I_{1}\right|=0
$$

and hence,

$$
\varlimsup_{\tau \rightarrow 0} \mid \tau^{-1}(H(t+\tau)-H(\tau)-F(t) \mid<\varepsilon .
$$

For $\tau<0$,

$$
\begin{aligned}
I_{2}-I_{3}= & \int_{0}^{\delta-\tau} \varphi(t+\tau-p)\left\{E\left(p^{-1 / 2}\right)-E\left((p-\tau)^{-1 / 2}\right\} d p\right. \\
& +\tau^{-1} \int_{0}^{\tau} \varphi(t-p) E\left(p^{-1 / 2}\right) d p \\
= & J_{1}^{\prime}+J_{2}^{\prime} .
\end{aligned}
$$


The estimates for $J_{2}^{\prime}$ are exactly like those of $J_{2}$; the only change in $J_{1}^{\prime}$ occurs in the range, $0 \leqq t_{1}<t_{2} \leqq \delta-\tau$, over which $M^{\prime}(\delta, \tau)$ is taken, but if $|\tau|<\delta$, then we strengthen the inequalities if we extend the range to $0 \leqq t<t_{2} \leqq 2 \delta$ and everything goes as before.

Thus $(d / d t) H(t)=F(t)$ and the lemma follows by easy comparison with (43).

The lemma established, we return to (42) where we now see that

$$
M^{-1} N_{1}=\left[\begin{array}{cc}
-2 S^{-1 / 2} & 2 C^{-1 / 2} \\
2 \sqrt{2} \widetilde{C}^{-1 / 2}-2 S^{-1 / 2} & 2 \sqrt{2} \widetilde{S}^{-1 / 2}+2 C^{-1 / 2}
\end{array}\right] .
$$

But

$$
\sqrt{2}\left\{\begin{array}{l}
C \\
S
\end{array}\right\}^{-1 / 2}=\widetilde{C}^{-1 / 2} \mp \widetilde{S}^{-1 / 2}
$$

so that

$$
M^{-1} N_{1}=2\left[\begin{array}{rr}
-S^{-1 / 2} & C^{-1 / 2} \\
C^{-1 / 2} & S^{-1 / 2}
\end{array}\right]
$$

and (42) becomes

$$
\left[\begin{array}{l}
f_{1} \\
g_{1}
\end{array}\right]=\left[\begin{array}{l}
C_{1} \\
D_{1}
\end{array}\right]+2\left[\begin{array}{rr}
-S^{-1 / 2} & C^{-1 / 2} \\
C^{-1 / 2} & S^{-1 / 2}
\end{array}\right]\left[\begin{array}{l}
f_{1} \\
g_{1}
\end{array}\right]
$$

Further, if we multiply the second equation in (44) by $i$ and add it to the first with

$$
\begin{aligned}
& f=f_{1}+i g_{1} \\
& a=C_{1}+i D_{i},
\end{aligned}
$$

(44) becomes

$$
f=a+2 i E^{-1 / 2} \bar{f}
$$

or

$$
f(t)=a(t)-\int_{0}^{t} k(t-s) \bar{f}(s) d s
$$

where

$$
k(t)=i \pi^{-1 / 2} e^{i \pi / 4} t^{-3 / 2} \exp (i / t) .
$$

Starting from system (40) we obtain in an analogous fashion

$$
g=b-2 i E^{-1 / 2} \bar{g}
$$

where $g=f_{2}+i g_{2}, b=C_{2}+i D_{2}$. Or, 


$$
g(t)=b(t)+\int_{0}^{t} k(t-s) \overline{g(s)} d s
$$

where $k$ is defined in (47).

These two problems are combined in the equation

$$
f(t)=a(t)+\lambda k * \bar{f}(t) .
$$

5. Solution of the integral equation. We here solve the integral equation (50) collecting our results at the end in the form of a theorem. By formal successive substitution of (50) into itself, we obtain

$$
f=a+\lambda k * \bar{a}+|\lambda|^{2} k *(\bar{k} * a)+\lambda|\lambda|^{2} k *(\bar{k} *(k * \bar{a}))+\cdots .
$$

In Appendix A, we show that for functions $a(t) \in \mathrm{CBV}[0, T]$ we can interchange the order of integration to obtain

$$
k *(\bar{k} * a)=(k * \bar{k}) * a=k_{2} * a
$$

where by explicit evaluation

$$
k_{2}(t)=(2 / \pi)^{1 / 2} t^{-3 / 2} \exp (-2 / t) .
$$

This function has the same form as $k$ (it is even absolutely integrable) so that again

$$
k *(\bar{k} *(k * \bar{a}))=k *\left(\bar{k}_{2} * \bar{a}\right)=\left(k * \bar{k}_{2}\right) * \bar{a} .
$$

Thus, we obtain for (51)

$$
f=a+\lambda k * \bar{a}+|\lambda|^{2} k_{2} * a+\lambda|\lambda|^{2} k_{3} * \bar{a}+\cdots
$$

where

$$
\begin{aligned}
k_{2 n} & =(2 / \pi)^{1 / 2} n t^{-3 / 2} \exp \left(-2 n^{2} / t\right) \\
k_{2 n+1} & =-\frac{1}{2}(2 / \pi)^{1 / 2}(2 n+1-i) t^{-3 / 2} \exp (-[n(2 n+2)-i(2 n+1)] / t)
\end{aligned}
$$

(see Appendix B for the computation).

To see that this series has meaning, one observes that on $[0, T]$

$$
\left|k_{2}\right| \leqq M_{2} \quad \text { and } \quad\left|k_{3}\right| \leqq M_{3}
$$

whence

$$
\left|k_{2 n}\right|=M_{2}^{n} t^{n-1} /(n-1) !, \quad n>0
$$

and

$$
\begin{aligned}
\left|k_{2 n+1}\right| & \leqq \int_{0}^{t}\left|k_{3}(t-s)\right|\left|k_{2(n-1)}(s)\right| d s \\
& \leqq M_{3} M_{2}^{n-1} t^{n-1} /(n-1) !, \quad n>0 .
\end{aligned}
$$


Hence, if $a \in \mathrm{CBV}$ and one sets $A=\max (a(t) ;[0, T])$ then

$$
\begin{aligned}
|f| \leqq & |\alpha|+|\lambda||k * \bar{a}| \\
& +|\lambda| A\left(M_{2}+|\lambda| M_{3}\right) \sum_{n=1}^{\infty}\left(|\lambda| M_{2}\right)^{n-1} T^{n-1} /(n-1) !
\end{aligned}
$$

which shows that (52) is a uniformly convergent series for all $\lambda$ and

$$
f=a+\lambda k * \bar{a}+\Sigma|\lambda|^{2 n}\left(k_{2 n} * a+\lambda k_{2 n+1} * \bar{a}\right) .
$$

To show that $f$, defined by (53), is continuous, one need only show that $k * \bar{a}$ is continuous; the other terms have very smooth kernels. If

$$
g(t)=\int_{0}^{t} s^{-3 / 2} \exp (i / s) a(t-s) d s,
$$

then for $t_{1}>t_{2}$

$$
\begin{aligned}
g\left(t_{1}\right)-g\left(t_{2}\right)= & \int_{t_{2}}^{t_{1}} s^{-3 / 2} \exp (i / s) a\left(t_{1}-s\right) d s \\
& +\int_{0}^{\delta} s^{-3 / 2} \exp (i / s) a_{2}(s) d s \\
& +\int_{\delta}^{t_{2}} s^{-3 / 2} \exp (i / s) a_{2}(s) d s \\
= & I_{1}+I_{2}+I_{3}
\end{aligned}
$$

where $a_{2}(s)=a\left(t_{1}-s\right)-a\left(t_{2}-s\right)$ and $\delta<(1 / 2) t_{1}$ is to be chosen. Now

$$
\left|I_{i}\right| \leqq(2 \sup |a|+2 V(a ;[0, T])) M_{i}, \quad i=1,2 .
$$

The existence of the integral implies that

$$
M_{1}=\sup \left|\int_{u}^{v} s^{-3 / 2} \exp (i / s) d s\right|, \quad t_{2} \leqq u<v \leqq t_{1}
$$

goes to zero as $t_{2} \rightarrow t_{1}$ and that

$$
M_{2}=\sup \left|\int_{u}^{v} \cdots\right|, \quad 0 \leqq u<v \leqq \delta,
$$

is small if $\delta$ is small. The uniform continutity of a makes $I_{3}$ small as $t_{2} \rightarrow t_{1}$.

One observes that

$$
\begin{aligned}
\lambda k * \bar{f}= & \lambda k * \bar{a}+|\lambda|^{2} k * \overline{(k * \bar{a})} \\
& +\lambda k * \overline{\left[\Sigma|\lambda|^{2 n}\left(k_{2 n} * a+\lambda k_{2 n+1} * \bar{a}\right)\right]} \\
= & -a+f,
\end{aligned}
$$

and we have proved: 
Theorem 4. If $a \in \mathrm{CBV}[0, T]$, then $f$, defined by (53), is a continuous solution of the equation

$$
f=\alpha+\lambda k * \bar{f}
$$

for all $\lambda$.

6. Solution for the finite problem. The function $f$, determined by (53), may not be BV; for example set $a(t)=1$. Thus, the solution of the system (34) may not satisfy the conditions of Theorems 1 and 2. However, we can establish the validity of the results directly.

Proposition. If a is $\mathrm{CBV}$, then

$$
U(x, t ; k * a)=-U(x+2, t ; a) .
$$

The proof of this proposition is easily effected by an inversion of the order of integration and evaluation of the interior integral. Justification of the interchange can be obtained by adapting the argument of Appendix A.

From (53) $f-\lambda k * \bar{a}$ is CBV, $a$ is CBV by assumption and the remaining sum has a bounded first derivative because of the form of $k_{m}, m \geqq 2$. Therefore,

$$
U(x, t ; f)=U(x, t ; f-\lambda k * \bar{a})-\lambda U(x+2, t ; \bar{a}),
$$

and from Theorem 2

$$
\lim _{\substack{x \rightarrow 0 \pm \\ t \rightarrow t_{0}}} U(x, t ; f)=-e^{i \pi / 4} I^{1 / 2}[f-\lambda k * \bar{a}]+\lambda E^{1 / 2} \bar{a} .
$$

We observe that $k * \bar{a}=-2 i E^{-1 / 2} \bar{a}$ and $-2 E^{-1 / 2}=i I^{-1 / 2} \widetilde{E}^{1 / 2}$ (the lemma) so that

$$
U(x, t ; f) \rightarrow-e^{i \pi / 4} I^{1 / 2} f .
$$

Also, from Theorem 2

$$
\lim _{\substack{x \rightarrow 0 \pm \\ t \rightarrow t_{0}}} U_{x}= \pm(f-\lambda k * \bar{a})-\lambda U_{x}(2, t ; \bar{a}) .
$$

Since $U_{x}(2, t ; \bar{a})=-k * \bar{a}$, we see that $U_{x}(x, t ; f) \rightarrow f(t)$ as $x \rightarrow 0+$ which is the only case of interest.

We thus conclude that if $I^{-1 / 2} a_{i}$ and $b_{i}(i=1,2)$ have first derivatives which are CBV then (33) provides a solution to the equation $\left(\partial_{x}^{4}+\partial_{t}^{2}\right) u=0$ with homogeneous initial conditions and boundary values given by (32).

Appendix A. To solve the integral equation above, it was neces- 
sary to interchange the order of integration for a very singular integrand. We here prove that the interchange is valid.

If $f$ is $\operatorname{CBV}[0, T]$ and $|a|>0,|b|>0, \operatorname{Re} a \geqq 0, \operatorname{Re} b \geqq 0$, then for $0 \leqq t \leqq T$

$$
\begin{aligned}
J_{1}= & \int_{0}^{t} \int_{0}^{s}[(t-s)(s-u)]^{-3 / 2} \exp \left(-a(t-s)^{-1}-b(s-u)^{-1}\right) \\
& \cdot x f(u) d u d s \\
= & \int_{0}^{t} f(u) \int_{u}^{t}\left[(t-s)(s-u)^{-3 / 2} \exp \left(-a(t-s)^{-1}-b(s-u)^{-1}\right)\right. \\
& \cdot x d s d u=J_{2} .
\end{aligned}
$$

The method of proof will be to show that $J_{2}$ exists, to restrict the domain to that on which interchange is easily justified, and then to show that the neglected terms vanish in the limit which will establish the existence of $J_{1}$ and the equality $J_{1}=J_{2}$.

The first integral of $J_{2}$ is evaluated explicitly in Appendix B as

$$
\sqrt{\pi}\left(\frac{1}{\sqrt{b}}+\frac{1}{\sqrt{a}}\right)(t-u)^{-3 / 2} \exp \left[-\frac{(\sqrt{a}+\sqrt{b})^{2}}{t}\right] .
$$

Thus

$$
J_{2}=C_{1} \int_{0}^{t}(t-u)^{-3 / 2} \exp \left(-c(t-u)^{-1}\right) f(u) d u
$$

which is equivalent to the first integration of $J_{1}$.

Now then,

$$
\begin{aligned}
I(s) & =\int_{0}^{s}(s-u)^{-3 / 2} \exp \left(-b(s-u)^{-1}\right) f(u) d u \\
& =\int_{0}^{s} u^{-3 / 2} \exp (-b / u) f(s-u) d u
\end{aligned}
$$

will exist if we can show that

$$
I(m, n)=\int_{m}^{n} u^{-3 / 2} \exp (-b / u) f(s-u) d u \rightarrow 0 \quad \text { as } n, m \rightarrow 0 .
$$

But

$$
I(m, n) \leqq(B+V(f,[0, T])) M(m, n)
$$

where $B=\sup |f(t)|, 0 \leqq t \leqq T$, and

$$
M(m, n)=\sup \left|\int_{w}^{z} u^{-3 / 2} \exp (-b / u) d u\right|, \quad m \leqq z \leqq n .
$$

The existence of the integral implies that $M(m, n) \rightarrow 0$ as $m, n \rightarrow 0$; hence, $I(s)$ exists. 
Moreover, similar estimates give (A.3)

$$
|I| \leqq(B+V(f,(0, s))) M(0, s),
$$

where by integration by parts

$$
\begin{aligned}
M(0, s) \leqq & |b|^{-1}\left|\exp (-b / z) z^{1 / 2}-\exp (-b / w) w^{1 / 2}\right| \\
& +\frac{1}{2}|b|^{-1}\left|\int_{w}^{z} u^{-1 / 2} d u\right|,
\end{aligned}
$$

and thus $M(0, s) \leqq 3|b|^{-1} s^{-1 / 2}$. Hence, $I(s)$ is well behaved near the origin so that the outside integral of $J_{1}$ is not improper at the origin and its existence will be established if

$$
J_{1}^{\prime}=\int_{\delta}^{t-\delta}(t-s)^{-3 / 2} \exp \left(-a(t-s)^{-1}\right) I(s) d s
$$

has a limit as $\delta \rightarrow 0$. However,

$$
\begin{aligned}
J_{1}^{\prime}= & \int_{\delta}^{t-\delta} s^{-3 / 2} \exp (-a / s) I(t-\delta-s) d s \\
& +\int_{\delta}^{t-\delta} s^{-3 / 2} \exp (-a / s)[I(t-s)-I(t-\delta-s)] d s
\end{aligned}
$$

where the first integral is proper and we may interchange the order of integration to obtain

$$
\begin{gathered}
\int_{0}^{t-2 \delta}\left(\int_{u}^{t}-\int_{u}^{u+\delta}-\int_{t-\delta}^{t}\right) \cdots d s d u \\
=J_{2}^{\prime}-I_{1}-I_{2} .
\end{gathered}
$$

Substitution into $J_{1}^{\prime}$ gives $J_{1}^{\prime}=J_{2}^{\prime}-I_{1}-I_{2}+I_{3}$, where $I_{3}$ is the second integral in $J_{1}^{\prime}$ above. Since $J_{2}$ exists, $\lim _{\delta \rightarrow 0} J_{2}^{\prime}=J_{2}$. We have only to show that $I_{i} \rightarrow 0(i=1,2,3)$ with $\delta$ to have $J_{1}=J_{2}$.

We integrate the first integral of $I_{3}$ by parts to obtain

$$
\begin{aligned}
& I(t-s)-I(t-\delta-s)=b^{-1} \exp (-b / \delta) \delta^{1 / 2} f(s-\delta) \\
& \quad-\frac{1}{2} b^{-1} \int_{0}^{s} u^{-1 / 2} \exp (-b / u) f(s-u) d u \\
& \quad+b^{-1} \int_{s-\delta}^{s}(s-u)^{1 / 2} \exp \left(-b(s-u)^{-1}\right) d f(u)
\end{aligned}
$$

which substituted into $I_{3}$ gives

$$
I_{3}=b^{-1}\left(I_{31}-I_{32}+I_{33}\right) \text {. }
$$

If we extend $f$ to the negative reals by $f(t)=f(0)$ for $t<0$, then

$$
I_{31}=b^{-1} \delta^{1 / 2} \exp (-b / \delta) \int_{\delta}^{t-\delta} s^{-3 / 2} \exp (-a / s) f(s-\delta) d s .
$$


The integral converges to $I(s)$ as $\delta \rightarrow 0$ so that $I_{31} \rightarrow 0$ as $\delta \rightarrow 0$. Observe that

$$
\left|\int_{0}^{\delta} u^{-1 / 2} \exp (-b / u) f(s-u) d u\right| \leqq[B+V(f)] M_{1}(0, \delta)
$$

where

$$
M_{1}(0, \delta)=\sup \left|\int_{w}^{z} u^{-1 / 2} \exp (-b / u) d u\right|, \quad 0 \leqq w<z \leqq \delta .
$$

By the same analysis as used to estimate $M(0, s)$ we see that $M_{1}(0, \delta) \leqq$ const. $\delta^{3 / 2}$ and hence

$$
\left|I_{32}\right| \leqq K \delta^{3 / 2} \int_{\delta}^{t-\delta} s^{-3 / 2} d s=K \delta^{3 / 2}\left(\delta^{-1 / 2}+(t-\delta)^{-1 / 2}\right) .
$$

Finally we have

$$
\left|\int_{s-\delta}^{s}(s-u)^{1 / 2} \exp \left(-u(s-u)^{-1}\right) d f(u)\right| \leqq K \delta^{1 / 2} V(f ;(s-\delta, s)) .
$$

Since $f$ is uniformly continuous on $[0, T]$ so is $V(f ;(0, s))$; thus, $V(f ;(s-\delta, s))=o(1)$ as $\delta \rightarrow 0$ uniformly in $s$, and

$$
\left|I_{33}\right| \leqq K \delta^{1 / 2} o(1) \int_{\delta}^{t-\delta} s^{-3 / 2} d s=o(1) .
$$

Integrals $I_{1}$ and $I_{2}$ are essentially the same as can be seen by substituting $s=u+v$ into $I_{1}$ and $s=t-v$ into $I_{2}$. We thus consider only $I_{1}$ which we write as

$$
I_{1}=\int_{0}^{t-2 \delta} f(u) \int_{0}^{t}[(t-u-v) v]^{-3 / 2} \exp \left(-a(t-u-v)^{-1}-b / v\right) d v d u .
$$

Integrating the first integral by parts (integrating $\exp (-b / v)$ ), we obtain

$$
\begin{aligned}
I_{1}= & b^{-1} \delta^{-1 / 2} \exp (-b / \delta) \int_{\delta}^{t-\delta} u^{-3 / 2} \exp (-a / u) f(t-u) d u \\
& -b^{-1} \int_{0}^{t-2 \delta} d u f(u) \int_{0}^{\delta} \exp \left(-b / v-a(t-u-v)^{-1}\right) \\
& \times\left[\frac{1}{2} v^{-1 / 2}(t-u-v)^{-3 / 2}+3 / 2 v^{1 / 2}(t-u-v)^{-5 / 2}\right. \\
& \left.-a v^{1 / 2}(t-u-v)^{-7 / 2}\right] d v,
\end{aligned}
$$

$I_{1}=b^{-1}\left(I_{11}-I_{12}-I_{13}+I_{14}\right)$. Since $I_{11}$ is just $I_{31}, I_{11} \rightarrow 0$ as $\delta \rightarrow 0$ Since $t-u-v \geqq \delta$, hence $(t-u-v)^{-\alpha} \leqq \delta^{-\alpha}(\alpha>0), I_{1 j}(j=$ $2,3,4)$ are absolute integrable as double integrals so that Fubini's 
theorem is applicable. Reversing the order of integration in $I_{12}$ we obtain

$$
\begin{aligned}
I_{12}= & b^{-1} \int_{0}^{\delta} \frac{1}{2} v^{-1 / 2} \exp (-b / v) \int_{0}^{t-2 \delta}(t-u-v)^{3 / 2} \exp \left(-a(t-u-v)^{-1}\right) \\
& \cdot x f(u) d u d v .
\end{aligned}
$$

The inside integral can be estimated by $[B+V(f)] M_{3}(\delta, v)$ where

$$
M_{3}(\delta, v)=\sup \mid \int_{w}^{z}(t-u-v)^{-3 / 2} \exp \left(-a(t-u-v)^{-1} d u \mid,\right.
$$

$0 \leqq w<z \leqq t-2 \delta$. By integration by parts we see that $M_{3}$ satisfies

$$
M_{3}(\delta, 0) \leqq 3|a|^{-1}(t-v)^{1 / 2}+|a|^{-1}(2 \delta-v)^{1 / 2} ;
$$

thus, $I_{12} \rightarrow 0$ as $\delta \rightarrow 0$. Similarly the inside integral $I_{13}$, after reversing the order of integration, can be estimated by $K\left[(2 \delta-v)^{-1 / 2}+(t-v)^{-1 / 2}\right]$ so that $I_{13} \rightarrow 0$ as $\delta \rightarrow 0$.

Estimates like those above are too coarse for $I_{14}$; we must take another approach. If we reverse the order of integration and then integrate the inner integral by parts

$$
\begin{aligned}
a I_{14}= & f(0) \int_{0}^{\delta} v^{1 / 2}(t-v)^{-3 / 2} \exp \left(-b / v-a(t-v)^{-1}\right) d v \\
& -f(t-2 \delta) \int_{0}^{\delta} v^{1 / 2}(2 \delta-v)^{-3 / 2} \exp \left(-b / v-a(t-v)^{-1} d v\right. \\
& +\int_{0}^{\delta} v^{1 / 2} \exp (-b / v) \int_{0}^{t-2 \delta} \exp \left(-a(t-u-v)^{-1}\right) \\
& \times\left[-3 / 2(t-u-v)^{-5 / 2} f(u) d u+(t-u-v)^{-3 / 2} d f(u)\right] d v ;
\end{aligned}
$$

that is

$$
a I_{.4}=f(0) I_{141}-f(t-2 \delta) I_{142}+I_{143}+I_{144} .
$$

Now, $I_{143}$ is just $I_{13}$ and

$$
\left|I_{141}\right| \leqq(t-\delta)^{-3 / 2} \int_{0}^{\delta} \sqrt{v} d v
$$

so that $I_{141}$ and $I_{143} \rightarrow 0$ as $\delta \rightarrow 0$.

For $I_{144}$ we have immediately that

$$
\left|I_{144}\right| \leqq \int_{0}^{\delta} v^{1 / 2} \int_{0}^{t-2 \delta}(t-u-v)^{-3 / 2} d V(f ;(0, u)) d v .
$$

If $\delta<1$ and $0<p<1, y=\delta^{p}>\delta$ and 


$$
\begin{aligned}
\int_{0}^{t-2 \delta}(t- & \left.u-v)^{-3 / 2} d V(f ; 0, u)\right)=\left(\int_{0}^{t-2 y}+\int_{t-y 2}^{t-\delta 2}\right)(t-u-v)^{-3 / 2} d V(f) \\
\leqq & (2 y-v)^{-3 / 2} V(f ;(0, t))+(2 y-v)^{-3 / 2} V(f ;(t-2 y, \xi)) \\
& +(2 \delta-v)^{-3 / 2} V(f ;(\xi, t-2 \delta))
\end{aligned}
$$

by the second mean value theorem for Riemann-Stieltjes integrals where $t-2 y \leqq \xi \leqq t-2 \delta$. Therefore,

$$
\left|I_{144}\right| \leqq 2 \delta^{3 / 2}(2 y-\delta)^{-3 / 2} V(f ;(0, t))+\frac{2}{3} V(f ;(\xi, t-2 \delta)) .
$$

Since $V(f,(0, u)$ is continuous in $u$, we have

$$
\left|I_{144}\right| \leqq 2 \delta^{3(1-p) / 2}\left(2-\delta^{1-p}\right)^{-3 / 2} V(f ;(0, t))+o(1)
$$

as $\delta \rightarrow 0$.

To complete the proof we have only to show that $I_{142} \rightarrow 0$ as $\delta \rightarrow 0$. The only question arises if $a$ and $b$ are both imaginary for which we write $b=i \beta, \alpha=i h \beta$. If we set $v=2 \delta /(w+1)$, than

$$
I_{142}=\left(\int_{1}^{N}+\int_{N}^{\infty}\right)(w+1)^{-1} w^{-3 / 2} \exp \left[-i \beta(w+1)(h w+1)(2 \delta w)^{-1}\right] d w .
$$

Given $\varepsilon>0$, we can choose $N$ such that

$$
\int_{N}^{\infty} \cdots d w \leqq \int_{n}^{\infty}(w+1)^{-1} w^{-3 / 2} d w \leqq 2(N+1)^{-1} N^{-1 / 2}<\varepsilon .
$$

The remaining integral goes to zero with $\delta$ by a stationary phase argument.

Appendix B. We here evaluate certain integrals which we have used. From [1] p. 146(28)

$$
L\left\{t^{-3 / 2} \exp \left(-\frac{1}{4} a / t\right)\right\}=2 \sqrt{\pi} \alpha^{-1 / 2} e^{-\sqrt{\alpha} s}
$$

for $\operatorname{Re}(\alpha)>0$. One can easily show that the formula is valid if $\operatorname{Re}(\alpha) \leqq 0,|\alpha| \neq 0$. Thus, using this formula we have

$$
\begin{aligned}
& \int_{0}^{t}[(t-u) u]^{-3 / 2} \exp \left(-a(t-u)^{-1}-b / u\right) d u \\
& \quad=\sqrt{\pi}\left(a^{-1 / 2}+b^{-1 / 2}\right) t^{-3 / 2} \exp \left[-\left(a^{1 / 2}+b^{1 / 2}\right)^{2} t^{-1}\right], \\
& \quad|\arg a| \leqq \pi / 2, \quad|\arg b| \leqq \pi / 2 .
\end{aligned}
$$

Thus for $k * \bar{k}, a=e^{i \pi / 2} b=e^{-i \pi / 2}$ and $k_{2}(t)=(2 / \pi)^{1 / 2} t^{-3} / 2 \exp (-2 / t)$.

$$
k_{3}(t)=k * \bar{k}_{2}=-\frac{1}{2} \sqrt{(2 / \pi)}(3-i) \exp \left[-(4-3 i) t^{-1}\right] .
$$


Finally, by induction

$$
k_{2 n}(t)=(2 / \pi)^{1 / 2} n t^{-3 / 2} \exp \left(-2 n^{2} / t\right)
$$

and hence

$$
\begin{aligned}
k_{2 n+1}(t) & =k * \bar{k}_{2 n} \\
& =-\frac{1}{2}(2 / \pi)^{1 / 2}(2 n+1-i) t^{-3 / 2} \exp \left(-[n(2 n+2)-i(2 n+1)] t^{-1}\right) .
\end{aligned}
$$

The author expresses his gratitude to Professor W. Fulks who suggested the problem and gave invaluable aid.

\section{REFERENCES}

1. A. Erdélyi et al., Tables of integral transforms, vol. 1, McGraw-Hill, New York, 1954.

2. E. W. Hobson, Theory of functions of a real variable, vol. 1, Dover, New York, 1957.

3. Bruno Pini, Contributi allo studio dell'equazione delle vibrazioni della sbarra elastica, Atti del Sem. Mat. Fis. Dell'Univ. Modena 8 (1958-59), 90-120.

Received September 2, 1966. Based on the author's Ph. D. thesis submitted to Oregon State University.

Stevens Institute of Technology

HobOKEN, NeW JeRSEY 


\section{PACIFIC JOURNAL OF MATHEMATICS}

\section{EDITORS}

\section{H. SAMELSON}

Stanford University

Stanford, California

J. P. JANS

University of Washington

Seattle, Washington 98105
J. Dugundji

University of Southern California Los Angeles, California 90007

RICHARD ARENS

University of California

Los Angeles, California 90024

\section{ASSOCIATE EDITORS}
E. F. BECKENBACH
B. H. NeumanN
F. WOLF
K. YosidA

\section{SUPPORTING INSTITUTIONS}

UNIVERSITY OF BRITISH COLUMBIA

CALIFORNIA INSTITUTE OF TECHNOLOGY

UNIVERSITY OF CALIFORNIA

MONTANA STATE UNIVERSITY

UNIVERSITY OF NEVADA

NEW MEXICO STATE UNIVERSITY

OREGON STATE UNIVERSITY

UNIVERSITY OF OREGON

OSAKA UNIVERSITY

UNIVERSITY OF SOUTHERN CALIFORNIA
STANFORD UNIVERSITY

UNIVERSITY OF TOKYO

UNIVERSITY OF UTAH

WASHINGTON STATE UNIVERSITY

UNIVERSITY OF WASHINGTON

AMERICAN MATHEMATICAL SOCIETY CHEVRON RESEARCH CORPORATION TRW SYSTEMS

NAVAL ORDNANCE TEST STATION 


\section{Pacific Journal of Mathematics \\ Vol. 22, No. $1 \quad$ January, 1967}

Charles A. Akemann, Some mapping properties of the group algebras of a

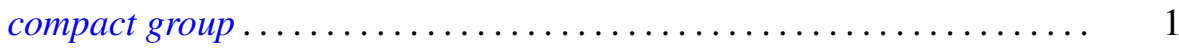

A. V. Boyd, Note on a paper by Uppuluri ..................... 9

Thomas Craig Brown, A semigroup union of disjoint locally finite subsemigroups which is not locally finite .....................

Richard Thomas Bumby and Everett C. Dade, Remark on a problem of

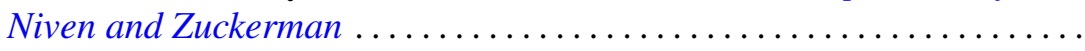

James Calvert, An integral inequality with applications to the Dirichlet problem .........................................

Jack Gary Ceder and Terrance Laverne Pearson, On products of maximally resolvable spaces ....................................

William Guignard Faris, The product formula for semigroups defined by

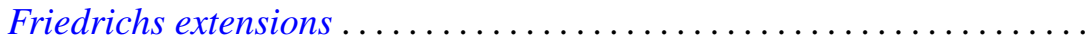

Robert S. Freeman, Closed operators and their adjoints associated with elliptic differential operators ........................ 71

Thomas Lee Hayden, The extension of bilinear functionals ............. 99

Gloria Conyers Hewitt, Limits in certain classes of abstract algebras . . . . . 109

Tilla Weinstein, The dilatation of some standard mappings ........... 117

Mitsuru Nakai, On Evans' kernel ......................... 125

Ernest Levane Roetman, On the biharmonic wave equation ............ 139

Malcolm Jay Sherman, Operators and inner functions ... . .

Walter Laws Smith, On the weak law of large numbers and the generalized elementary renewal theorem

A. J. Ward, On H-equivalence of uniformities: The Isbell-Smith problem 